\title{
Man sollte ständig Walnüsse futtern
}

\section{Hier steht eine Anzeige.}

\section{Die Substitution der Nahrung mit täglich $43 \mathrm{~g}$ Walnüssen führt zu einer signifikanten Senkung von LDL-Cholesterin- und Triglyzeridwerten.}

_ Die in Walnüssen enthaltenen Omega-3-Fettsäuren schützen Herz und Gefäße. In den bisherigen Studien wurden die Nüsse additiv zu anderen modifizierten Diäten eingesetzt. Bislang ist nicht ganz klar, ob es für den lipidsenkenden Effekt eine Rolle spielt, welche Nährstoffe durch die Walnüsse isokalorisch ersetzt werden.

In einer randomisierten, kontrollierten, prospektiven Cross-over-Studie an 194 gesunden Probanden im Alter von $63 \pm 7$ Jahren untersuchte man nach einer nussfreien Vorlaufperiode zwei Diätphasen von je acht Wochen Dauer. 96 Probanden nahmen zuerst täglich

$43 \mathrm{~g}$ Walnüsse zu sich und wechselten nach einer Washout-Periode von vier Wochen zur nussfreien Diät. 98 Personen durchliefen die Diätformen in umgekehrter Reihenfolge. Zusätzlich wurden die Probanden während der Walnussperiode in Gruppen mit Kohlenhydratreduktion, Fettreduktion oder beidem randomisiert.

Während der Walnuss-Diät verringerte sich der NüchternCholesterinwert um 8,5 \pm 37,2 und der LDL-Cholesterinwert um 7,4 $\pm 32,4 \mathrm{mg} / \mathrm{dl}$. In den Kontrollgruppen fiel die Verringerung signifikant niedriger aus $(1,1 \pm 35,4$ bzw. $1,7 \pm 29,7 \mathrm{mg} / \mathrm{dl})$. Auch die Triglyzerid- und Apolipoprotein-B-Spiegel nahmen unter der Walnussdiät signifikant ab. Interessanterweise spielten weder die Reduktion von Fett oder Kohlenhydraten noch der Zeitpunkt der Zufuhr von Walnüssen für den Lipidstatus eine Rolle.

- Bamberger C, Rossmeier A, Lechner K et al. A walnut-enriched diet reduces lipids in healthy Caucasian subjects, independent of recommended macronutrient replacement and time point of consumption: a prospective, randomized, controlled trial. Nutrients. 2017;9:E1097

\section{KOMMENTAR}

Der interessanteste Aspekt dieser Studie ist, dass es egal war, ob die Probanden ihre Fettzufuhr reduzierten oder nicht. Zwar geben die Autoren zu, dass trotz intensiver Diätberatung die diätetische Compliance der Probanden zu wünschen übrig ließ und dass die Nahrungszusammensetzung nur über ein Diättagebuch kontrolliert wurde. Doch kann man das auch als Real-world-Situation betrachten. Für die praktische Umsetzung ist besonders ermunternd, dass man die Walnüsse nach Belieben über den Tag verteilt konsumieren kann.

Prof. Dr. med. H. S. Füeß|

\author{
Springer
}

